\title{
Influencia de la ubicación de las aberturas en la eficiencia de la ventilación en viviendas
}

\author{
Effect of openings location on the ventilation efficiency in \\ dwellings
}

Alberto Meiss ${ }^{(*)}$, Jesús Feijó(*)

\section{RESUMEN}

El proceso de ventilación incluye impulsar aire "limpio" y extraer el viciado a través de los cerramientos, distribuir y hacer circular el aire entrante y prevenir la contaminación interior. El Código Técnico de la Edificación, en su Sección HS3 - Calidad del Aire Interior, establece unos caudales mínimos de ventilación generalistas a cumplir en viviendas en función de su tipología, superficie, posibles fuentes contaminantes y número de ocupantes, sin considerar la trayectoria del aire desde su admisión hasta su extracción. Por dicha razón ocurre el hecho que, aunque se cumpla la Normativa en cuanto al recinto a ventilar, pueden existir zonas en el interior que presenten exceso o déficit de ventilación.

El estudio que se presenta tiene como objetivo superar el cumplimiento meramente cuantitativo del DB-HS3, para controlar cualitativamente la renovación del aire en todo el espacio habitable, lo que permite incluso reducir el caudal con el consiguiente ahorro energético.

$313-5$

Palabras clave: Ventilación, eficiencia de renovación del aire, calidad del aire interior, edad del aire, ahorro energético

\section{SUMMARY}

Building ventilation implies managing the "clean" air and extracting the contaminated air through the walls, distributing and circulating the incoming air and preventing the contamination of the indoor air. The Spanish Technical Building Code, in his section HS3 - IAQ, usually set up minimum ventilation flow rates according to its typology, surface, possible contamination sources or the number of occupants, without thinking over the path of the air from the inlet to the exhaust point. For that reason, even if such standards are met within the spaces of the home, there may still be areas in the interior that suffer an excess or deficit of ventilation.

The present study aims to overcome the purely quantitative performance of $D B$ $H S 3$, to control qualitatively the air renewal throughout the living space; this allows even to reduce the volumetric flow rate with the consequent energetic saving.

Keywords: Ventilation, air change efficiency, indoor air quality, age-of-the-air, energy savings 
1. Sistema de ventilación en funcionamiento.

2. Consumo de energía primaria por sectores productivos.

\section{INTRODUCCIÓN}

La calidad del aire en ambientes interiores es un componente esencial para el bienestar de sus ocupantes, por lo que la ventilación es una de las instalaciones que más incidencia tiene en la salud y en el confort de dichos espacios. Las personas de los países industrializados pasan más del $90 \%$ de su tiempo dentro de ambientes artificiales tales como oficinas, fábricas, edificios públicos, viviendas, vehículos de transporte, etc. (1). Por esta razón se comprueba con preocupación que el progreso tecnológico, que está mejorando los estándares de las viviendas en general, no se corresponde con el deterioro que se está produciendo en la calidad del aire interior de las mismas.

Como concepto, la ventilación en los edificios suministra la cantidad requerida de aire hacia el interior, bajo unas condiciones climáticas y ambientales determinadas. El proceso incluye impulsar aire limpio y extraer el viciado a través de los cerramientos, distribuir y hacer circular el aire entrante y prevenir la contaminación interior.
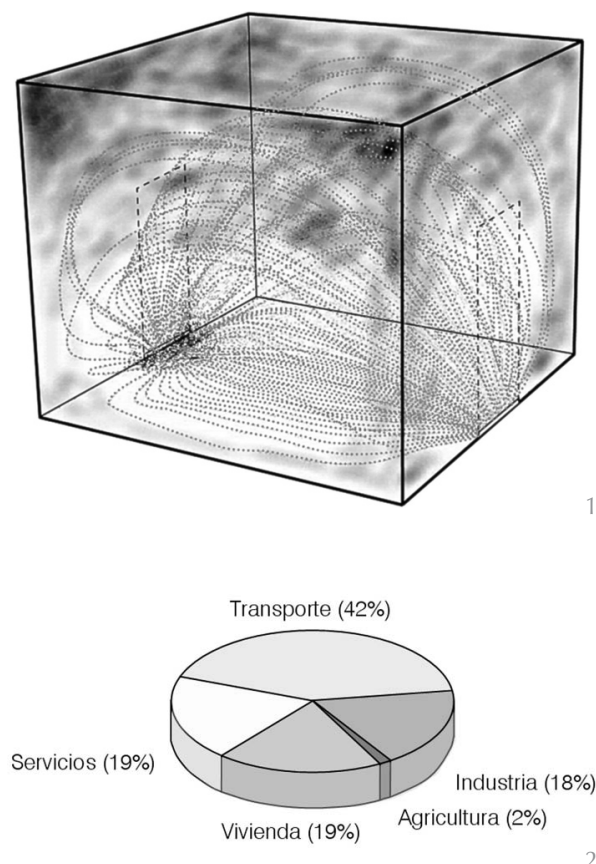

Las distintas normativas nacionales vigentes suelen estudiar los locales de manera global, es decir, estableciendo unos caudales mínimos de ventilación en función de su tipología, superficie, posibles fuentes contaminantes o número de ocupantes, tal es el caso del DBHS3 Calidad del aire interior. Sin embargo, aunque se cumpla dicha normativa en el recinto, pueden existir zonas en su interior que presenten exceso o déficit de ventilación.

El problema se ilustra en la Figura 1 en la cual se observa la simulación de un sistema de ventilación en funcionamiento: el aire penetra a través de la holgura inferior de una puerta y abandona el local a través de una abertura similar situada enfrente. Se puede observar que se crean grandes zonas de aire estancado, sin renovar, lo que indica que sólo una pequeña proporción del aire suministrado se dispersa por el local; esto evidencia de forma cualitativa que este sistema no es eficiente a la hora de "proveer aire a aquellas zonas de la habitación que lo requieran", aún cumpliendo incluso con el aporte del caudal mínimo normativo.

Por este motivo es necesario analizar la eficiencia de la ventilación de manera pormenorizada: dicho procedimiento dará lugar a estudios que permitan optimizar los sistemas utilizados y, al funcionar más eficientemente, conseguir un importante ahorro energético.

\subsection{El impacto energético de la ventilación}

Sucede que el correcto diseño de los sistemas de ventilación tiene un impacto significativo sobre el consumo de energía: según datos de la OCDE (2), el consumo de energía asociado con los edificios (residenciales y de servicios) representa alrededor del $40 \%$ del total de energía primaria consumida (Figura 2), en donde la ventilación y las pérdidas por infiltración representan una proporción importante. A partir de estos datos, utilizar una estrategia de ventilación más eficiente equivale a actuar para reducir el consumo sobre el 5 9\% del total de energía primaria usada en el planeta (3).

\section{METODOLOGÍA}

Independientemente cómo sea el sistema de impulsión de la ventilación (natural, híbrido o mecánico), la ubicación física de las aberturas de admisión y extracción de aire inciden significativamente sobre la eficiencia de renovación del aire de un recinto, $\varepsilon^{a}$, entendida esta como la capacidad del sistema para asegurar la adecuada renovación del aire contenido en el volumen, eliminando la polución existente en su interior (4). Cuando se presenta el supuesto que la fuente contaminante no está localizada y se desea a la vez asegurar la adecuada calidad del aire en todo el volumen de la habitación, la eficiencia de renovación del aire $\varepsilon^{a}$ permite un análisis genérico de los locales y de sus sistemas de ventilación, siendo el coeficiente utilizado en este trabajo de investigación (5).

La eficiencia de renovación del aire $\varepsilon^{a}$ se define como la relación entre el menor tiempo posible que toma reemplazar el aire, o tiempo de renovación mínimo $t_{t}$, y el tiempo medio que tarda en hacerlo, o tiempo medio de renovación del aire $\bar{\tau}_{\text {exc }}$ que es igual a dos veces la edad media del aire de la habitación [1]: 
$\varepsilon^{a}=\frac{\tau_{\mathrm{t}}}{\bar{\tau}_{\mathrm{exc}}} \cdot 100$

$[1]$

En los ensayos experimentales la eficiencia $\varepsilon^{a}$ se calcula dividiendo la edad media puntual del aire en la extracción $\tau_{\text {e }}$ por dos veces la edad media del aire de la habitación $\langle\bar{\tau}>$ (ya que tomamos como referencia el modelo óptimo, el Ilamado "flujo de pistón") [2]:

$\varepsilon^{a}=\frac{\tau_{e}}{2 \cdot\langle\bar{\tau}\rangle} \cdot 100$

Determinada en la fase de diseño de la vivienda, la disposición de las aberturas suele ser decisión del arquitecto, sin que éste sea consciente de la importancia que tienen sobre el movimiento del flujo de aire. Es, por tanto, fundamental aportar al proyectista herramientas rápidas y fiables que le permitan conocer a priori las consecuencias directas que tienen sus decisiones sobre la eficiencia de renovación del aire del recinto diseñado.

\subsection{Bases del procedimiento experimental y numérico}

Disponer de una cámara de ensayos en el Laboratorio de Ventilación HS3 de la Escuela de Arquitectura de Valladolid ha permitido llevar a cabo un exhaustivo estudio experimental del flujo del aire en distintas configuraciones de habitaciones, mediante el uso de gases trazadores. El caso aquí expuesto es la réplica de un dormitorio con sus correspondientes dispositivos de entrada y salida del aire: el primero mediante una abertura de admisión directa en consonancia con la carpintería exterior de la habitación, y el otro caracterizado, como suele suceder, con la holgura inferior de la hoja de la puerta (Figura 3).

Se establecieron unas condiciones de contorno para esta fase de la investigación:

- no se contempla la apertura de puertas o ventanas, ni cualquier otra causa que produzca un régimen no estacionario (el objeto es analizar la eficiencia cumpliendo las condiciones mínimas de ventilación);

- se impone un valor cero en la edad media puntual del aire en la impulsión al recinto;

- se presupone que el desplazamiento del aire es perpendicular a los cerramientos, con velocidad uniforme y cuyo valor es el obtenido según el caudal impuesto por normativa ( $10 \mathrm{l} / \mathrm{s}$ para un dormitorio de 2 ocupantes, según el DB-HS3);

- el régimen es isotermo, sin diferencias de temperatura entre el flujo de aire entrante

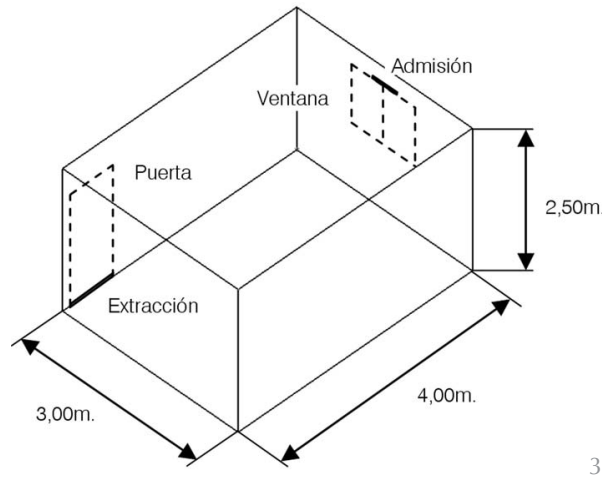

3. Modelo utilizado en los ensayos experimentales.

4. Localización de los puntos de validación. y el contenido dentro de la habitación. El resto de superficies que conforman el volumen se consideran paredes lisas e isotermas.

Mediante mediciones realizadas en 18 puntos de control se caracteriza el flujo del aire desde que entra hasta que abandona la habitación. Estos 18 puntos son el resultado de los nueve que aparecen en planta, con mediciones realizadas en dos alturas distintas, a 0,75 y $2,00 \mathrm{~m}$ sobre el nivel del pavimento (Figura 4).

El estudio consistió de 73 ensayos según el método de la caída de la concentración del gas trazador, calculándose la edad del aire en cada uno de esos puntos (6) (todos en series de tres puntos, dos correspondientes a puntos de control y otro en consonancia

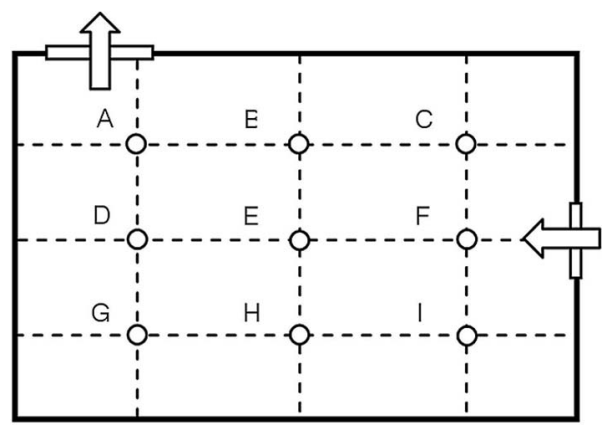

con la salida del aire, lo que permite adimensionalizar los resultados). Con la concentración de referencia, 125 mg de $\mathrm{SF}_{6} /$ $\mathrm{m}^{3}$ de aire, las desviaciones medias respecto a la media experimental se cuantifican en $+1,58 \%$ y $-1,65 \%$, con máximas puntuales de $+4,10 \%$ y $-6,52 \%$, lo que puede considerarse muy satisfactorio de cara a caracterizar el patrón de flujo existente. Estos valores son los que permitirán la validación del modelo numérico.

La principal ventaja de las técnicas CFD (Computational Fluid Dynamics) frente a las experimentales es la reducción sustancial de tiempo y coste. Además estas técnicas proporcionan gran cantidad de datos del 
5. Validación del modelo numérico a partir de los resultados experimentales.

6. Ejemplo de tabla de caracterización de los recintos a estudiar. modelo de flujo: distribuciones de velocidad, temperatura, edad del aire, satisfacción del usuario, etc. Sin embargo, dada la gran cantidad de variables de configuración (mallado, condiciones de contorno, modelos de turbulencia, resolución de ecuaciones, etc.), que condicionan la bondad de los resultados, es necesario la validación de los modelos numéricos con los datos obtenidos experimentalmente.

El estudio numérico ha sido desarrollado utilizando el software CFD Fluent 6.3.26 de la empresa ANSYS. La simulación de la habitación reproducida en la cámara de ensayos permite obtener las edades en los puntos de control, que se comparan con los obtenidos experimentalmente (Figura 5), lo que nos permite dar por buena la configuración adoptada en el estudio numérico para utilizarla posteriormente en el análisis de variantes alternativas de diseño.

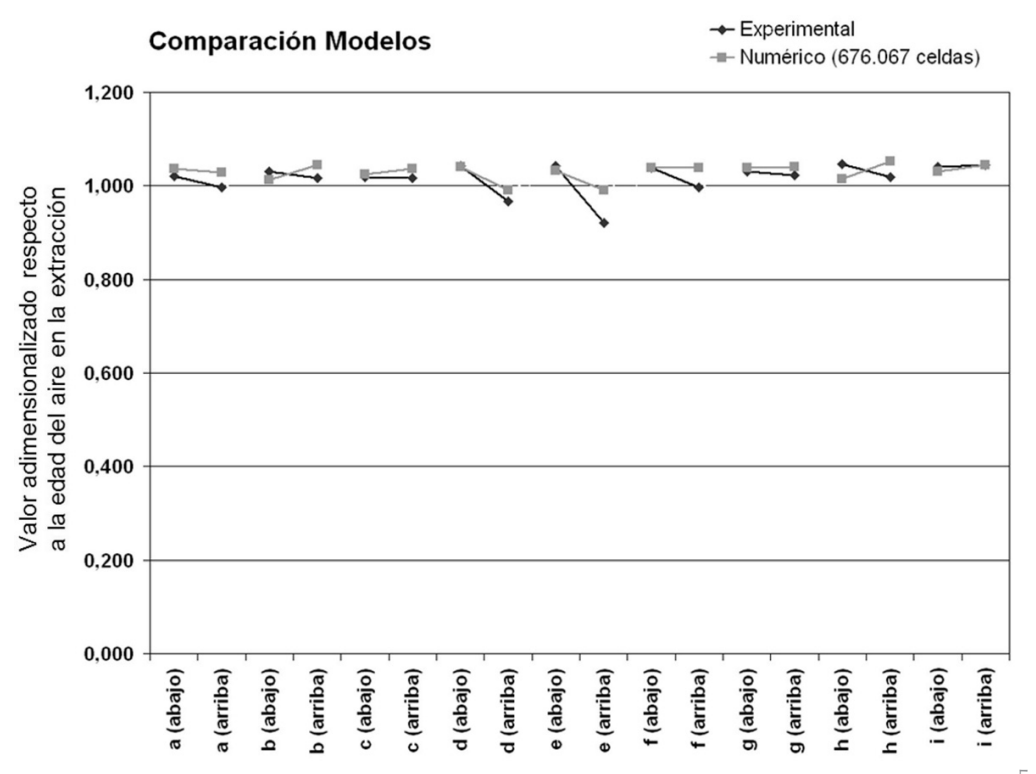

\begin{tabular}{|c|c|c|c|c|c|c|c|c|c|}
\hline & \multicolumn{2}{|c|}{ EFICIENCIA } & \multicolumn{3}{|c|}{ IMPULSIÓN } & \multicolumn{2}{|c|}{ EXTRACCIÓN } & \multicolumn{2}{|c|}{ RECNNTO } \\
\hline 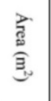 & 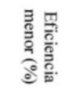 & 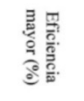 & 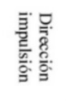 & 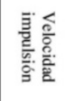 & $\boxminus$ & 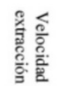 & 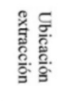 & 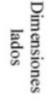 & 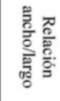 \\
\hline \multirow{2}{*}{ 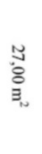 } & $40,81 \%$ & $57,20 \%$ & $\stackrel{8}{\sharp}$ & 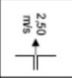 & D $73+D 74+D 75+D 76$ & 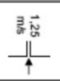 & $\square$ & \multirow[b]{2}{*}{ 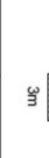 } & \\
\hline & $39,42 \%$ & $56,37 \%$ & $\stackrel{8}{i}$ & 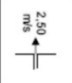 & $\mathbf{D} 77+\mathbf{D} 78+\mathbf{D} 79+\mathbf{D} 80$ & 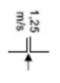 & $\square$ & & $\mu_{9}$ \\
\hline \multirow{2}{*}{ 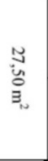 } & $44,39 \%$ & $55,62 \%$ & $f^{8}$ & $\begin{array}{c}\frac{y_{y}}{4} \\
\uparrow\end{array}$ & D81 & 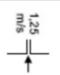 & $\square$ & \multicolumn{2}{|c|}{$\frac{0}{3}$} \\
\hline & $42,86 \%$ & $55,30 \%$ & $\stackrel{8}{i}$ & 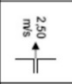 & D82 & 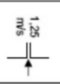 & $\square$ & \multicolumn{2}{|l|}{ 今 } \\
\hline 焉 & $47,85 \%$ & $51,82 \%$ & $\overbrace{}^{8}$ & 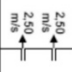 & D83+D84 & 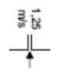 & $\square$ & $\square_{\text {wort }}^{\square_{3}^{g}}$ & \\
\hline 产 & $47,47 \%$ & $51,50 \%$ & $i^{8}$ & 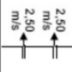 & D85+D86 & 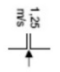 & $\square$ & 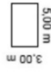 & \\
\hline
\end{tabular}

2.2. Estudio de la eficiencia de la ventilación en viviendas según la ubicación de las aberturas de admisión y extracción de aire (7)

En cada una de las habitaciones de la vivienda se produce un proceso de entrada y salida del aire de ventilación, estableciéndose un flujo cuya función es la renovación del aire del recinto, con la consiguiente eliminación de los contaminantes en él contenidos. La definición arquitectónica de los locales que integran una vivienda ventilada se hace básicamente a través de parámetros que involucran las dimensiones propias del recinto y la ubicación relativa y las características geométricas de las aberturas de ventilación presentes.

Este trabajo se ha desarrollado en condiciones isotermas, obviando la ocupación y el mobiliario. No obstante debe indicarse que la acción de la flotabilidad debida a flujos a distinta temperaturas se produce principalmente en los locales con aberturas de admisión de aire exterior (dormitorios y salas de estar) y en situaciones de invierno-verano, que en todo caso provocan mayor movimiento de aire, optimizando los resultados obtenidos. En todo caso, la tendencia normal del flujo es convertirse paulatinamente en isotermo respecto al resto del aire de la vivienda en su discurrir hacia la extracción.

Con la intención de abarcar la práctica totalidad de las viviendas que se construyen hoy día se han definido 156 morfologías de habitación desde aseos a cuartos de estar. Las variables que determinaron dicho número son:

- el volumen de los locales, con superficies entre 1,5 y $35 \mathrm{~m}^{2}$ útiles y altura libre entre 2,20 y 2,50 m;

- la forma del recinto, rectangular o irregular según la proporción entre los lados de la planta;

- el número de aberturas y su ubicación relativa;

- la velocidad del aire en las aberturas y el caudal circulante.

Como ejemplo se adjunta una de las múltiples tablas de caracterización de los recintos a estudiar (Figura 6).

En total se han realizado 1.152 procesos de cálculo de otras tantas variantes con programas de CFD (simulaciones numéricas), configuradas según las variables de los modelos base validados con ensayos experimentales en la cámara del laboratorio. Mediante este cálculo múltiple es posible determinar la influencia que tiene el disponer las aberturas en 
diferentes localizaciones, pudiéndose dibujar gráficos que indiquen la eficiencia obtenida según la ubicación de la extracción respecto a la impulsión de aire.

Ello nos ha permitido conocer el comportamiento de la eficiencia según el tamaño de la habitación y la ubicación de sus aberturas. Para mayor facilidad de comprensión, este comportamiento se ha trasladado a una representación gráfica de la planta de la habitación, fácilmente interpretable para aplicar en el diseño arquitectónico: los resultados se representan utilizando un código de colores (de 0 a $100 \%$ de eficiencia) que expresan el valor calculado para la ubicación relativa del elemento dentro de la habitación (Figura 7). De manera simplificada se puede decir que las eficiencias obtenidas pueden agruparse en cuatro grupos según sus valores:

- el primero es el menos eficiente y comprende valores iguales o menores de $40 \%$ tendentes al modelo de cortocircuito;

- el segundo es para mayores de $40 \%$ y menores o iguales a $50 \%$ que se puede considerar próximos al modelo de mezcla perfecta;

- el tercero para valores mayores de 50\% alcanzando un modelo de desplazamiento;

- y el cuarto, para valores superiores a $60 \%$, muy difíciles de conseguir en espacios convencionales, tendentes al modelo teórico de flujo pistón.

\subsection{Diseño y cálculo con ábacos (7)}

Tras calcular los múltiples locales de viviendas se observó que era posible agrupar los resultados para construir herramientas que fueran útiles al proyectista; en concreto ábacos que permitieran obtener de manera directa los valores de la eficiencia de la renovación del aire en el recinto considerado.

Los ábacos se construyeron para las diferentes disposiciones de aberturas de ventilación, según ejes referenciados en la admisión respecto a la extracción: la ordenada y expresa la distancia en profundidad entre las dos aberturas, y el eje de abscisas $\mathrm{x}$ indica la distancia lateral entre sus ejes. El valor en cada punto expresa la eficiencia $\varepsilon^{a}$ obtenida para dicha localización (Figura 8).

\section{EJEMPLO DE APLICACIÓN}

El DB HS-3 ha supuesto para España un revulsivo para la ventilación de viviendas, puesto

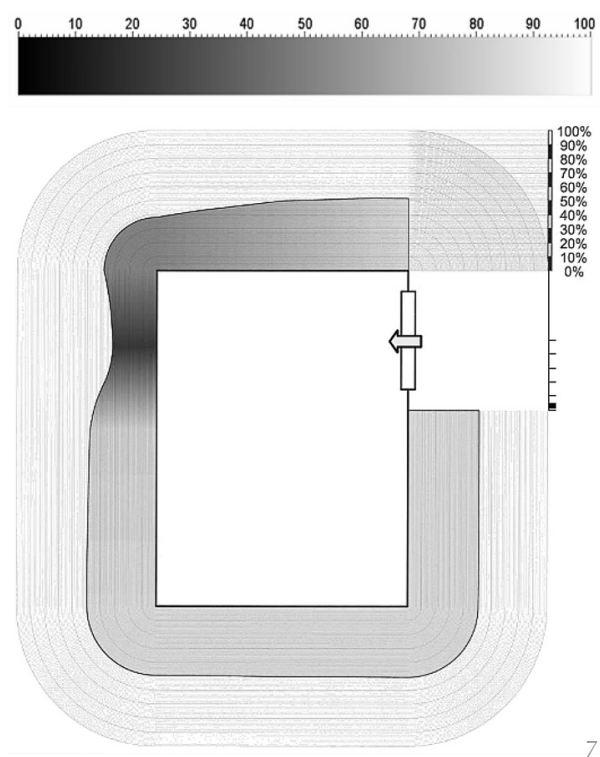

7. Escala según patrón de color para representar la eficiencia de la renovación del aire $\varepsilon^{a}$ en un recinto (en \%) y resultados obtenidos en un recinto dado.

8. Ábaco para determinar la eficiencia de la ventilación $\varepsilon^{a}$ en recintos con aberturas de ventilación situadas en la parte baja del local.

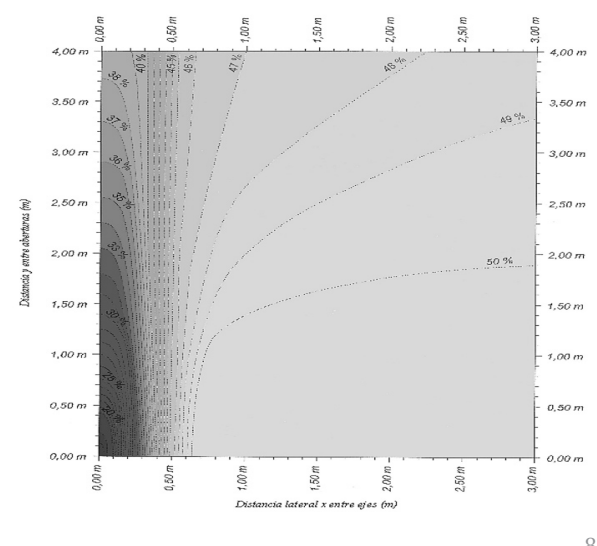

que se ha pasado de una práctica desregulación del tema a fijar unas condiciones mínimas y elementales, en sintonía con otros países de nuestro entorno, significando para nuestro marco normativo un salto cualitativo infinito. Por ese motivo los caudales establecidos son de una premeditada simplicidad previendo futuras perfecciones, entre otras la determinación del caudal mínimo según la forma del local y la disposición de sus aberturas.

Aunque por esa misma simplicidad no se explicite la eficiencia de la ventilación en el DB HS-3, los caudales que se demandan se basan en una eficiencia equivalente a un modelo de mezcla $\left(\varepsilon^{a}=50 \%\right)$, o lo que es lo mismo, el aire contenido en el recinto (edad media del aire de la habitación $<\bar{\tau}>$ ) deberá tener al menos la edad del aire extraído $\left(\tau_{\mathrm{e}}\right)$ : esto refleja que la salida de aire transporta los contaminantes producidos y almacenados en el interior y que el aire entrante ocupa su lugar. A continuación se plasman las tres posibilidades en cuanto a eficiencia se refiere.

$\varepsilon^{a}=\frac{\tau_{e}}{2 \cdot\langle\bar{\tau}\rangle}<50 \%$

El recinto no se renueva eficientemente $=$ mala calidad del aire interior 
9. Recinto de ejemplo: sala de estar de una vivienda.

10. Posibles ubicaciones distintas donde situar la admisión de aire al recinto (de 1 a 13)

11. Determinación de $\varepsilon^{a}$ según la distinta ubicación de la admisión.

12. Trayectoria del aire desde su entrada hasta su extracción según la ubicación de la admisión. $\varepsilon^{a}=\frac{\tau_{e}}{2 \cdot\langle\bar{\tau}\rangle}=50 \%$

Prescripciones mínimas del DB-HS3

$\varepsilon^{a}=\frac{\tau_{e}}{2 \cdot\langle\bar{\tau}\rangle}>50 \%$

Se puede reducir el caudal hasta $\varepsilon^{a}=50 \%$

(Lo exigido por normativa)

Un ejemplo concreto permite calibrar la importancia de estos conceptos, tanto en lo que significa determinar la eficiencia de la ventilación de los locales que se proyectan como para interpretar qué significa en cuanto a caudal necesario de ventilación.

Suponemos que se trata de una sala de estar de $27,00 \mathrm{~m}^{2}$ con planta asimilable a una "L" (Figura 9). Según el DB-HS3 se fija un caudal mínimo de ventilación de $3 \mathrm{l} / \mathrm{s}$ por
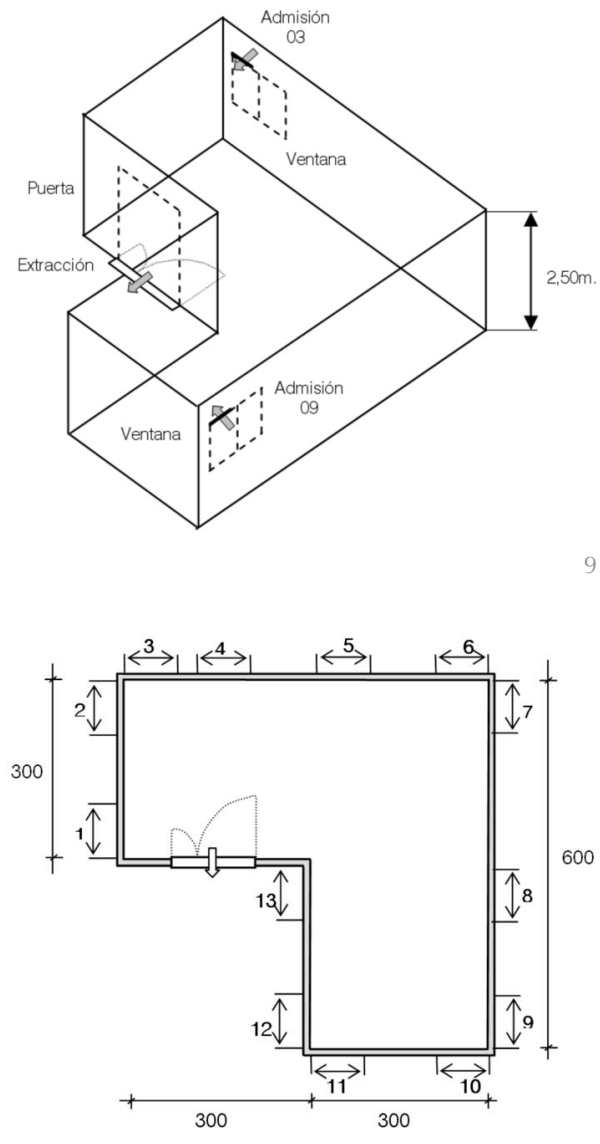

10

ocupante, cuyo número sería el mismo que el de las personas que duermen según tipo y número de dormitorios. Además, como es preceptivo, habría que hacer un equilibrado global entre admisiones y extracciones, con lo que nos llevaría a un caudal en el recinto de $20 \mathrm{l} / \mathrm{s}$ (o 1,07 renovaciones/hora) para cumplir las condiciones de calidad mínima del aire interior.
La admisión se plantea como una abertura ligada a las carpinterías exteriores de las ventanas y la extracción coincidente con la holgura inferior de la hoja de la puerta. Con estas premisas, podríamos definir razonablemente hasta 13 localizaciones distintas para plantear la entrada del aire. Definir las consecuencias de adoptar una u otra ubicación (Figura 10) es precisamente lo que se quiere valorar a efectos de ventilación.

A partir del procedimiento de cálculo expuesto anteriormente es posible determinar la eficiencia $\varepsilon^{a}$ según ubiquemos la admisión en los diferentes puntos (Figura 11).
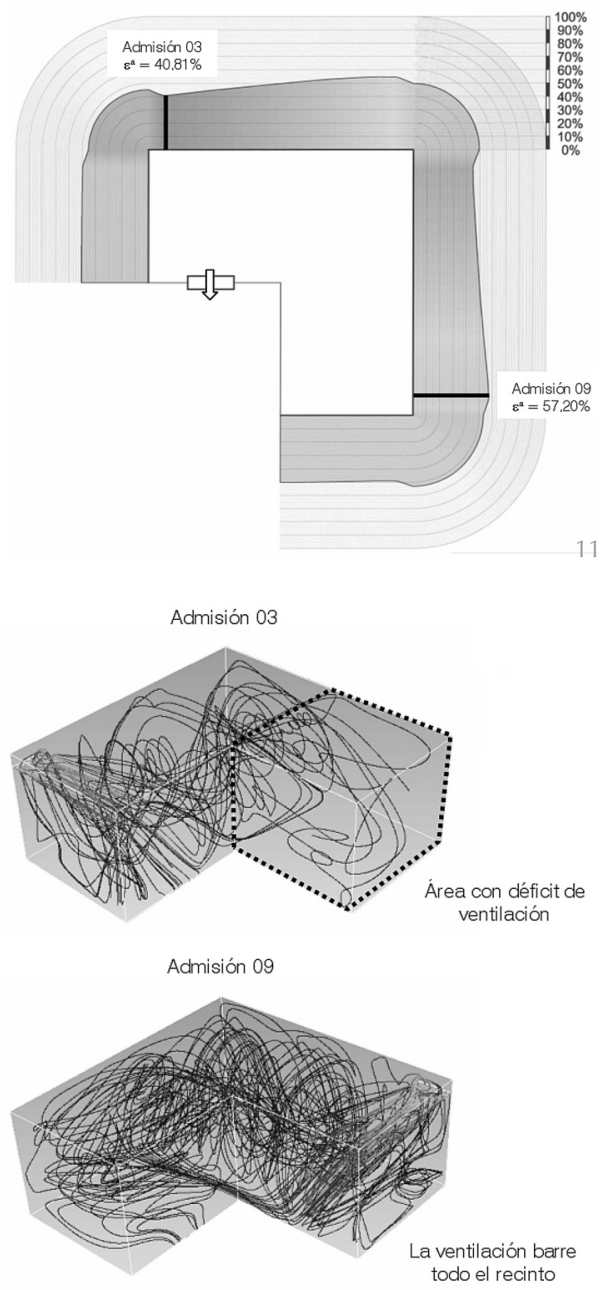

12

Si enfrentáramos la admisión de la ventana con la puerta de la sala (Admisión 03), su baja eficiencia $\left(\varepsilon^{a}=40,81 \%<50 \%\right.$ ) nos indica que deberíamos aumentar el caudal de ventilación hasta alcanzar una calidad de aire interior mínima para los ocupantes. Esto se aprecia estudiando el movimiento del aire y los valores resultantes de la edad en los distintos puntos del espacio (Figura 12): la mayor parte del aire entrante no transporta los contaminantes producidos y almacenados en el interior de la vivienda y es expulsado al exterior "relativamente limpio", con el 
consabido despilfarro energético. Por otro lado, amplias zonas del local son capaces de almacenar contaminantes hasta un grado tal que se superen los niveles aceptables para la salud humana.

La consecuencia es que, a pesar de cumplir con el caudal de ventilación preceptivo, no somos capaces de ventilar adecuadamente el espacio y la calidad del aire resultante es deficiente. La única solución sería aumentar el caudal de ventilación circulante (según cálculos desde los 20,00 l/s establecidos por el CTE, hasta los $23,80 \mathrm{l} / \mathrm{s}$, donde se obtiene una edad media $\langle\bar{\tau}\rangle=3.375 \mathrm{~s}$, equivalente a la eficiencia $\varepsilon^{a}=50 \%$, Figura 13$)$.

En cambio, cuando proyectamos recintos que permitan eficiencias superiores al 50\% puede reducirse significativamente el caudal necesario, al cumplir con la calidad mínima exigida al aire interior, hasta que se satisfaga la condición que la edad media $\langle\bar{\tau}\rangle$ del recinto iguale a la edad en la extracción $\tau_{e}$. En nuestro caso, si al proyectar la sala de estar de la vivienda dispusiéramos la admisión de manera que forzara un barrido de todo el ámbito del local (Admisión 09) podríamos reducir el caudal necesario de ventilación desde los 20,00 l/s hasta los $17,00 \mathrm{l} / \mathrm{s}$.

Esto equivale a un significativo ahorro energético, pues minimizamos las pérdidas que implica la entrada incontrolada de aire del exterior, así como una mejor sensación de confort interior (menor riesgo de corrientes de aire molestas, menor impacto acústico, etc.).

\section{CONCLUSIONES}

El arquitecto, al diseñar las viviendas, debe ser capaz de incorporar en su proyecto el concepto de la ventilación, modelando el flujo del aire de manera que discurra de la forma más eficiente desde que entra hasta que sale del local. Esto es muy importante para contribuir a asegurar una condición saludable del aire interior conjuntamente con un ahorro energético perceptible, en el caso de obtener eficiencias $\varepsilon^{a}$ mayores del $50 \%$. Para esto, el proyectista ha de disponer de herramientas que permitan cuantificar directamente el comportamiento del sistema proyectado, y que indiquen pautas que ayuden a un mejor diseño.

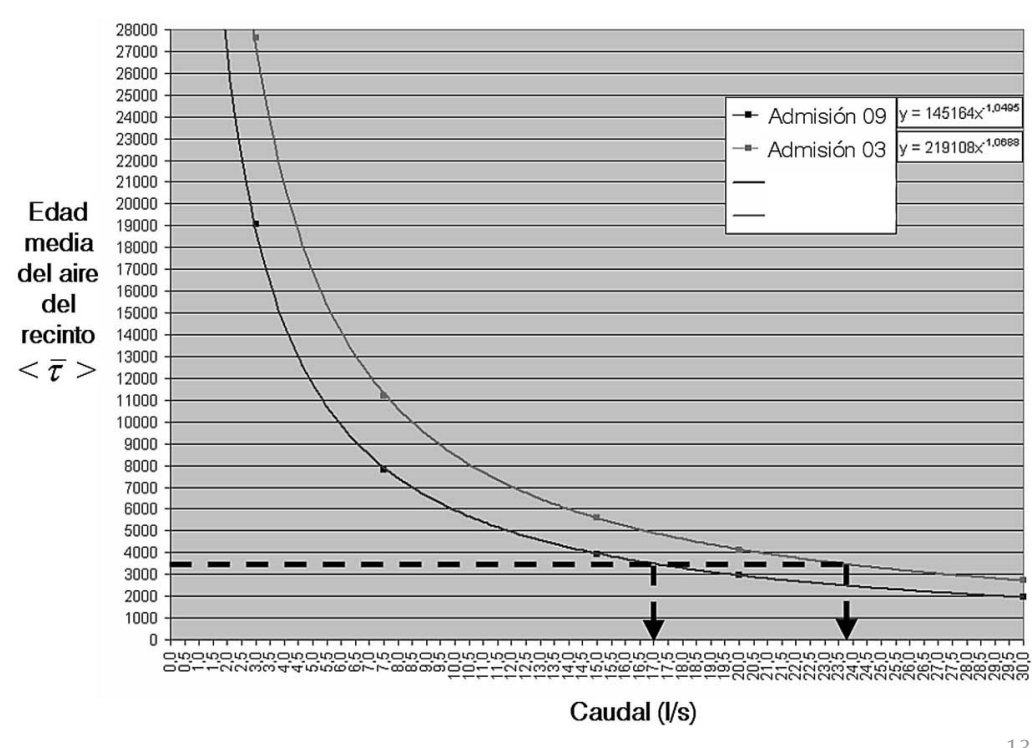

El proceso consiste en buscar las ubicaciones recomendables para las aberturas de admisión y extracción, en donde se observen mejores eficiencias en la renovación del aire del recinto, y diseñar el espacio a partir de ellas. En el caso que la disposición de dichas aberturas estuviese sujeta a restricciones que condicionaran el diseño, es posible buscar soluciones alternativas que minimicen la mala eficiencia del espacio proyectado (como cambiar la altura relativa de las aberturas, velocidades de admisión, ángulo de penetración del chorro de aire, etc.).

Teniendo en cuenta el gran impacto que tiene la ventilación residencial sobre el consumo energético mundial, la suma de pequeñas actuaciones en este sentido (a través de una optimización de la eficiencia del sistema) puede significar un considerable y perceptible ahorro en las economías del mundo desarrollado.

\section{AGRADECIMIENTOS}

La presente publicación forma parte del proyecto de investigación "Optimización de las aberturas de admisión del CTE-HS3 - Calidad del aire interior", financiado por el Ministerio de Educación y Ciencia de España (ref. ARQ2002-02177) con cofinanciación del FEDER, desarrollado en el período 2006-2009.
13. Relación caudal necesario / edad media del aire de la habitación $<\bar{\tau}>$. 


\section{BIBLIOGRAFÍA}

(1) Awbi, H.: Ventilation of buildings. p. 522, Spon Press. Londres, 1991.

(2) International Energy Agency IAE, Organisation for Economic Cooperation and Development: "Energy Balances for OECD Countries 1997-98". p. 82, París, 2000.

(3) Axley, J.: Residential passive ventilation systems: evaluation and design. p. 158, Air Infiltration and Ventilation Centre AIVC. Coventry, 2001.

(4) Feijó, J.; Camino, M.; Basterra, A.; Carbayo, J.: "Eficiencia de la ventilación en locales de extracción de viviendas". Proceedings of AICVF - Université de la Rochelle (2006), pp. 455-462.

(5) Mundt, E.; Mathisen, H-M.; Nielsen. P.; Moser A.: Ventilation effectiveness. Guidebook $\mathrm{n}^{\circ}$ 2. Rehva. p. 75, Federation of European Heating and Air-conditioning Associations. Helsinki, 2004.

(6) Sandberg, M.; Sjöverg, M.: "The use of moments for assessing air quality in ventilated rooms". Building and Environment. Vol. 18 (1983), pp. 181-197.

(7) Meiss, A.: Tesis doctoral "Estudio de la eficiencia de la ventilación en viviendas a partir de parámetros de diseño arquitectónico". p. 767, Universidad de Valladolid. Valladolid, 2009. 